\title{
Fostering microgeneration in power systems: The effect of legislative limitations ${ }^{\text {th }}$
}

\author{
J.N. Fidalgo ${ }^{a}$, Dalila B.M.M. Fontes ${ }^{\mathrm{b}, *}$ \\ a INESC-Porto, Faculdade de Engenharia, Universidade do Porto, R. Dr. Roberto Frias, 4200-465 Porto, Portugal \\ ${ }^{\mathrm{b}}$ LIAAD-INESC L.A., Faculdade de Economia, Universidade do Porto, R. Dr. Roberto Frias, 4200-464 Porto, Portugal
}

\section{A R T I C L E I N F O}

\section{Article history:}

Received 8 February 2011

Received in revised form

19 September 2011

Accepted 10 November 2011

Available online 5 December 2011

\section{Keywords:}

Microgeneration

Renewable energy

Regulatory framework

Distribution systems

\begin{abstract}
A B S T R A C T
The large-scale integration of microgeneration (MG) can bring several technical benefits, such as: improving the voltage profile, reducing power losses and allowing for network capacity investment deferral. Furthermore, it is now widely accepted that introducing new renewable MG, such as wind turbines, photovoltaic panels or biomass can help control carbon emissions, reduce our dependence on oil and contribute to a sustainable energy growth. This paper presents an empirical analysis of the benefits of MG on avoided losses, voltage profiles and branch congestion. The main goal is to clarify whether the current regulatory framework allows for obtaining all the MG potential gains. The main conclusion is that some legal constraints should be removed, or at least relaxed, in order to promote the growth of distributed power generation, particularly, for domestic MG.
\end{abstract}

(C) 2011 Elsevier B.V. All rights reserved.

\section{Introduction}

The international economic crisis and, in particular, the fluctuations in the price of fossil fuels has led to a search for alternative and greener energy sources. Furthermore, two other factors have contributed to the increase in investments in such technologies: environmental concerns and the prevailing European Union policy to secure the energy supply. The latter issue is mainly due to the strong dependency on imported energy sources. Many of these issues are dealt with on an international basis, for example the European Union (EU) outlines strategies and sets targets for its member states. However, energy policies are set domestically. Current environmental concerns and the instability of oil prices have increased the pressure on energy policy makers due to the increase in both energy imports and CO2 emissions. In September 2001, the EU proposed the Directive 077/CE/2001 on electricity production based on renewable sources.The Directive set the goal for renewable energy production at 39\% for Portugal by 2010 . A few years later Portugal established a new target of $45 \%$. The introduction of renewable energy sources is being encouraged in order to satisfy the goals set by the Kyoto agreement. Renewable energy sources are characterized by their decentralized production [1], thus a substantial proliferation of MicroGeneration (MG) is expected through

\footnotetext{
岤 The financial support of FCT Project PTDC/EGE-GES/099741/2008 is acknowledged.

* Corresponding author. Tel.: +351 225571241; fax: +351225505050

E-mail addresses: jfidalgo@inescporto.pt (J.N. Fidalgo), fontes@fep.up.pt (D.B.M.M. Fontes).
}

small hydro power generators, fuel cells, solar and wind power producers.

The current penetration growth of small MG is changing the paradigms associated with the exploitation, management and control of power systems. This trend is expected to continue to increase in Europe and it is being encouraged by the recent regulation directives that offer financial incentives for MG, offering a very advantageous context for individual consumers/producers. Furthermore, scientific literature shows that, generally, MG has a positive impact on power networks as it decreases losses, enhances voltage profiles and reduces branch congestion [2-6].

In 2008, as part of the Energy End-use Efficiency and Energy Services Directive (ESD), the Portuguese Government set the goal of installing $165 \mathrm{MW}$ of MG by 2015 [7,8]. The National Energy Efficiency Action Plan [7] includes several incentives that make MG more attractive to consumers/producers. These incentives include economic incentives with interesting tariff design whereby the MG owner is paid a rate of $650 € / M W h$ for photovoltaic energy, $455 € /$ MWh for wind power and $195 € /$ MWh for hydro energy, during the first 5 years. This is a key incentive to encourage investments in MG, since the reference energy price in Portugal at Low Voltage (LV) level is around $110 € /$ MWh.

These advantageous conditions are, however, bounded by the following constraints:

1. In order to maintain the quality of service in the energy supplied by the distribution network operator(DNO), the regulations stipulate that the MG penetration is must be limited to $25 \%$ of the 
secondary substation rated power and the onsite installed capacity should not exceed $50 \%$ of the contracted power;

2. To control the eventual impact of MG on tariffs, the subsidized tariff values mentioned above are only applicable for the first $10 \mathrm{MW}$ of installed capacity. Each additional $10 \mathrm{MW}$ increase will be penalized with a $5 \%$ decrease on the tariff's base reference.

The limitations imposed above in condition 1 aim to control the quality of service by adopting a conservative policy on the integration of MG and therefore, its implications are analyzed in this paper. The conditions described above in 2 have important consequences. Firstly, they promote the rapid growth of MG, since the first consumers/producers will be rewarded more generously. Secondly, the decreasing reward policy prevents excessive amounts of MG energy production being introduced into the system. The reason behind this conservative policy is the fear that high levels of MG could negatively affect operating conditions. The 2009 experiment shows that the first $10 \mathrm{MW}$ slot was promptly occupied, however, the number of new MG installations rapidly decreased and the second $10 \mathrm{MW}$ slot was not even completely used. This is most likely due to the penalties in the tariff rates for the later slots. This paper analyzes these constraints and discusses whether the second constraint should be removed, or at least relaxed.

Similar incentives are being adopted in most European countries and they are accompanied by the required legislative amendments to promote a favorable regulatory environment [9]. For instance, Sweden designed an attractive scheme of incentives for consumers for the installation of heat pumps [10]. Germany allocated 15 billion Euros for the development of photovoltaic technology. The British Department for Business, Energy and Regulatory Reform expects a growth in MG that would make it equivalent to five nuclear power stations [10]. The European Parliament's Subgroup Energy and Industry adopted "Microgeneration" and "improving energy awareness of consumers" as the new predominant keywords for their energy policy [11]. In the USA, some states are compelling utilities to incorporate a minimum percentage of energy generation from renewable sources. To meet such requirements, power companies are using transferrable "renewable source energy credits" in order to subsidize, or at least partially subsidize, renewable source microgeneration projects in their service areas [12].

The policy incentives that have been, and are still being implemented, suggest a remarkable growth of MG penetration in the distribution systems.

In the last decade researchers have taken an interest in MG. A considerable part of the scientific literature on this subject focuses on the impact of MG on the power networks [13-17]. These studies evaluate the changes in the system's quality parameters following the incorporation of MG into the network and illustrate the advantages of MG. Regulation aspects, as well as market issues and energy policies concerning MG, have also been studied [18-21].

The main conclusions drawn are that incentives should be provided to promote further integration of MG and that, although considering MG when planning new networks may lead to different optimal design policies, no additional investments are required to accommodate the integration of MG in existing networks. The impact of MG on the stability of power systems is analyzed in [22], where it has been shown that MG can improve the stability of power systems if suitable types of MG and appropriate locations are selected. In [23], the authors review the potential of MG for domestic demand-side management. A number of papers describe studies on the economic impact of the growth of MG, see [24-27].

This paper analyzes the technical impact of MG on a typical network and evaluates the adequacy of the limits that have been established. The technical impact indexes considered are: the active power losses, the voltage profiles and the branches load level. The main objective is to analyze whether, according to these variables the limitations on MG penetration are consistent with reality or not. In the latter case, the situation will be analyzed to ascertain if it is possible to alter these limitations and permit higher levels of integration of MG.

Section 2 of this paper presents the methodology, simulation scenarios, and the characterization of the simulated network. The results and conclusions are presented in Sections 3 and 4, respectively.

\section{Methodology}

Although regulators recognize the advantages of MG and stimulate its use, the constraints mean that the existing regulations are somewhat conservative, as stated in the introduction. The main issue behind this conservative attitude is the regulators' concern with the possible degradation of operation conditions. Therefore, in this work a wide range of scenarios relating to MG will be simulated to evaluate the technical impact on power systems. The impact of MG is characterized by the technical changes it causes, particularly on power and energy losses, voltage profiles and branch congestion.

The analysis of the current regulatory framework is performed in two phases:

1. Phase 1 - Intensive simulation of a variety of operational scenarios in order to obtain a implicit characterization of the effects of MG on the distribution network;

2. Phase 2 - Based on the results obtained in the previous phase, the adequacy of the MG regulation limits currently in place will be assessed.

The network analyzed in this study was provided by a leading Portuguese energy distribution company which considers it as typical - somehow representative of the Portuguese reality. The simulations are performed in a semi-urban medium voltage network. This network has a single injection point (bus 190) and feeds 115 load buses. The total typical load is about $6 \mathrm{MW}$.

In the simulations we have considered several scenarios which were obtained by using 7 load possibilities, 6 different MG locations, 2 dispersion settings and 21 levels of MG penetration. These include:

Load levels : varying from $20 \%$ up to $140 \%$ of the nominal value: C20, C30, C50, C80, C90, C100 and C140.

MG location distributions : Near the feeder (NF), far away from the feeder (FF), and 4 distinct randomly generated locations (RND1-RND4).

Dispersion settings : MG units are distributed over 12 and 25 buses (MG12 and MG25, respectively).

MG penetration levels : ranging from $0 \%$ to $100 \%$ in $5 \%$ intervals.

By varying the load level we intend to characterize how this parameter can affect the impact that MG has on the power system. Note that, for instance, a large MG value may be quite beneficial when load is high but disadvantageous when it is low. The MG location is also an important factor affecting the technical impact of MG on the network. Therefore, six different possibilities have been considered: near the feeder (NF), where the MG units are located near to the substation, far away from the feeder (FF), where the MG units are located far away from the substation, and RND1 RDN2, RDN3, and RND4, where the MG units are randomly distributed in the network. As shown in the results section, the power quality indexes may alter significantly depending on the MG distribution. Two dispersion distributions are considered with the purpose of analyzing whether a more concentrated MG distribution or a more wide-spread MG distribution is more advantageous. The MG effect 
is simulated through the reduction of the value of the load (active power) in the corresponding bus bar. The values of such reductions were chosen so that the percentage of MG ranges from $0 \%$ up to $100 \%$. For the lower limit, no MG exists, while for the upper limit all loads are fed by MG units and therefore no power is supplied by the substation. Although very high levels of integration are highly improbable, they have been included in order to fully assess the scenario.

\subsection{Algorithm}

A simulation platform was developed using MathPower to perform the power flow analysis and it has been used to analyze the network's behavior in each of the 1764 operational scenarios described above. This simulation platform will therefore analyze the impact on power losses, voltage profile and branch congestion. The simulation program considers the following steps sequentially:

1. Run the load-flow program (Newton-Raphson).

2. Evaluate branch active losses, branch currents intensities and bus voltages.

3. Aggregate the network total load.

4. Compute the network total losses.

5. Compute annual energy losses based on the results obtained in the previous step and on the typical load duration curve.

Power losses can be significant under heavy load conditions. With the inclusion of MG, power flows are decreased leading to a reduction of power losses in the distribution network. However, depending on the ratings and location of the MG units, power losses can increase at very high and unrealistic penetration levels, as shown in our results section. In this study, the voltage profile analysis is performed using the calculus of the mean voltage in the network. However, the voltage is monitored at each bus bar and, in case of voltage limit violations, the simulation is stopped, and the maximum level MG penetration is set to the previous valid point. In Portugal the regulation laws state that voltage should be kept within the range $[0.95 ; 1.10]$ p.u. Another beneficial technical impact is the reduction in line congestion, which typically allows for deferring investment in network capacity. In this study, the impact of MG is analyzed on the most loaded branch (relative to its capacity), in order to pinpoint the most sensitive section. The impact of MG is inferred by analyzing the load reduction in this branch.

\section{Results}

The simulations performed show the influence of MG on the network operating conditions. This is very important since the regulator must be aware of the technical impact when making decisions on possible regulation changes.

This section presents some of the results obtained from the simulations that were performed using the network mentioned above. Due to the large amount of outputted data, only a small selection of the main illustrative results is presented in this section.

\subsection{Power and energy losses}

Fig. 1 depicts the variation of the active power losses, for each considered load level, with the penetration of MG. The graphs show that MG generally contributes to a decrease in active losses. The graphs show the active power losses in percentage of power loss for C100 and without MG. In fact, the power losses decrease with MG penetration for all cases, for penetration levels below $50 \%$. For higher penetration levels, in the NF case and also when MG units distribution is concentrated (MG12), the losses start to increase,
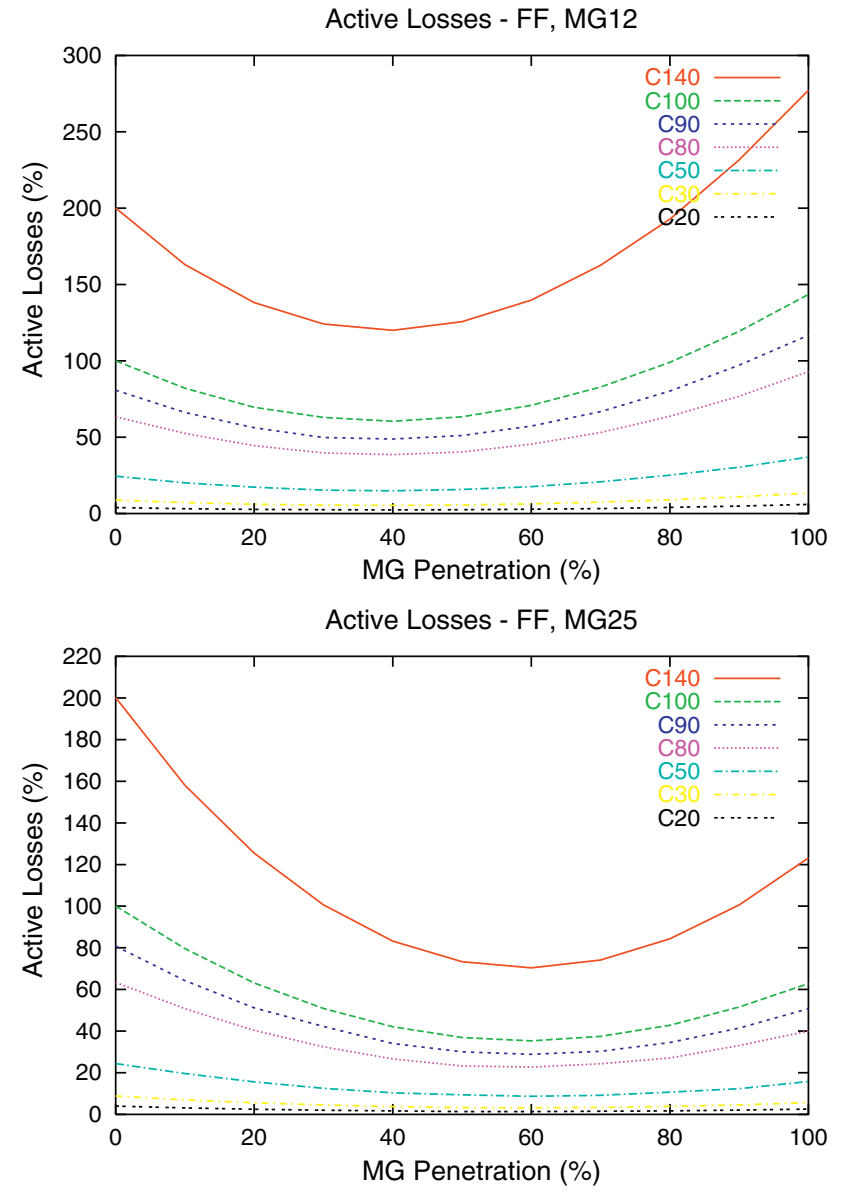

Active Losses - NF, MG25

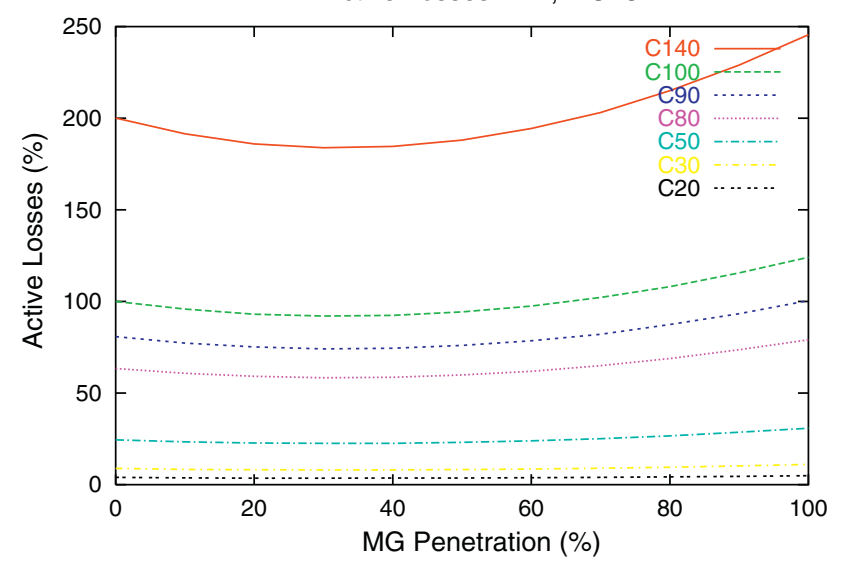

Fig. 1. Effects of MG dispersion and location on the active power losses.

indicating a power flow reversion in some branches. However, for the far away case if a wider dispersion of MG units is considered, power losses are always smaller regardless of MG penetration levels. Nevertheless, the maximum reduction is obtained for a MG penetration level of about $50 \%$.

The graph at the top shows the active losses for the far from the feeder (FF) scenario when the MG unit distribution is concentrated (MG12). The middle graph uses the same MG location scenario but with wider MG dispersion (MG25). The comparison between these two graphs leads to the conclusion that a larger dispersion of MG generally results in a larger reduction of active losses, especially for higher levels of integration of MG. The bottom graph represents the near to the feeders (NF) location scenario with wider 
Energy Losses - FF, MG12

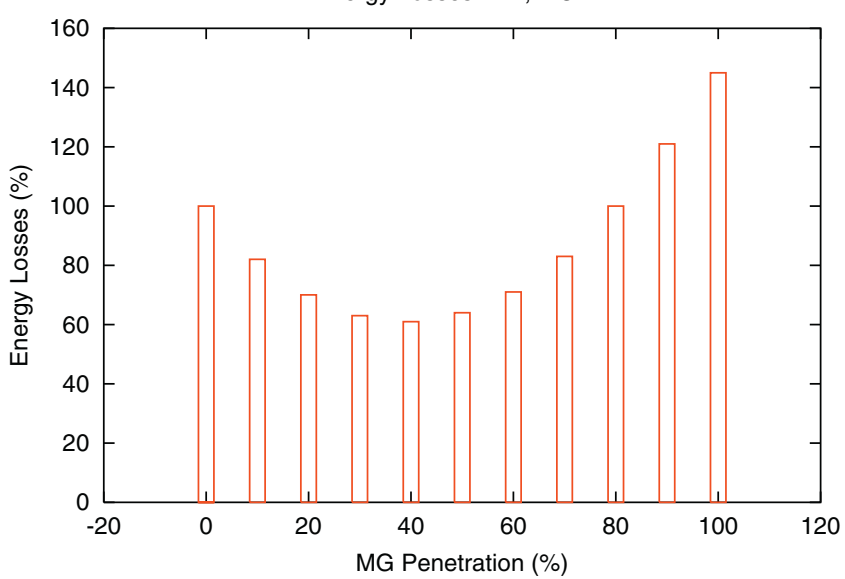

Energy Losses - FF, MG25

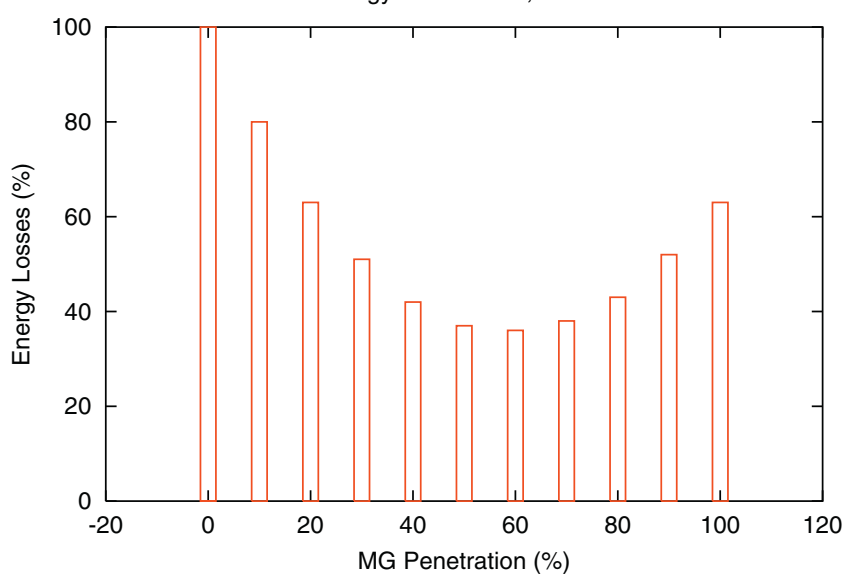

Fig. 2. Yearly energy losses.

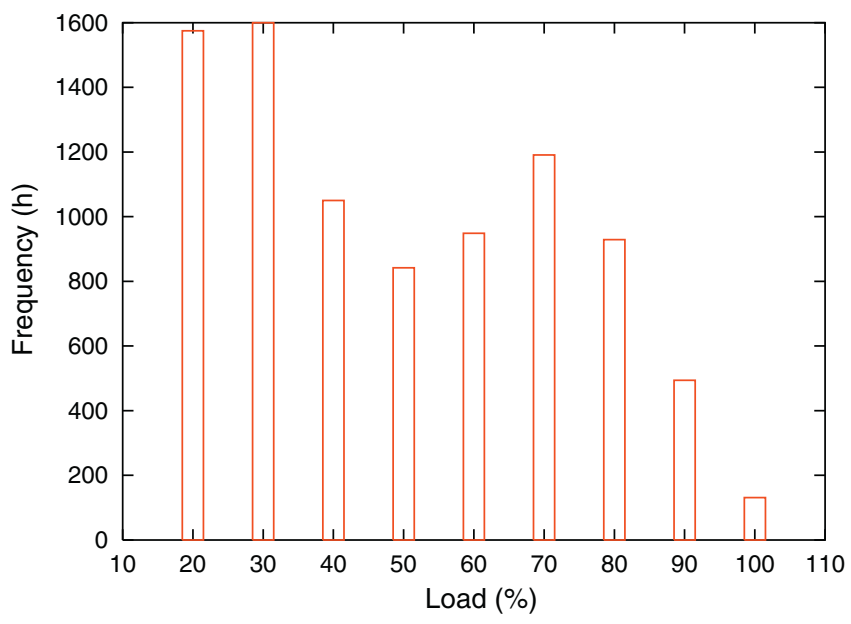

Fig. 3. Typical load duration curve.

dispersion (MG25) of the MG units. By comparing this graph with the middle graph, it can be concluded, unsurprisingly, that the system performs better if the MG units are placed far from the feeders (i.e. near loads).

The annual energy losses are shown in Fig. 2. The shape of the graph is comparable to the power loss shapes and the conclusions drawn for power loss analysis are also valid for the energy case. These losses were evaluated using the typical load duration curve shown in Fig. 3.

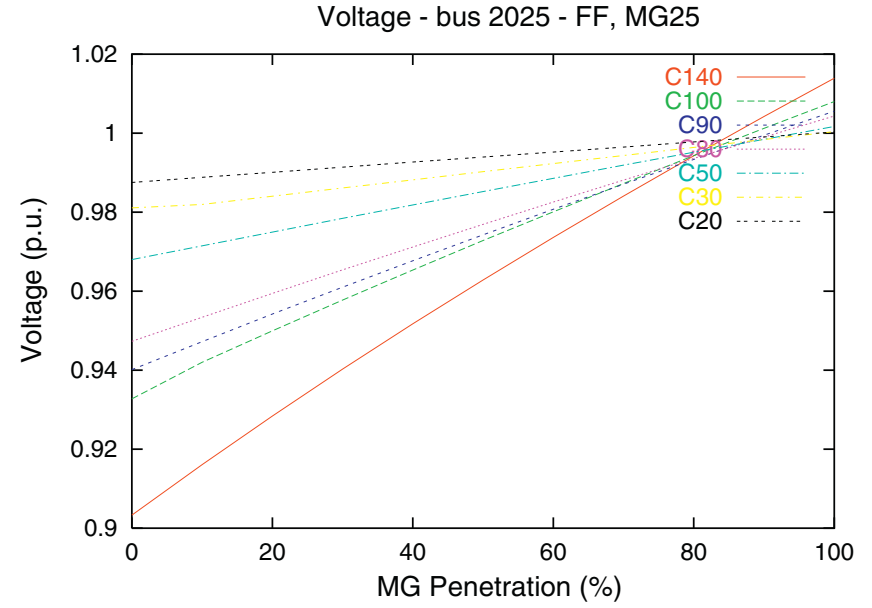

Fig. 4. Effects of the MG penetration level on bus voltage.

\subsection{Voltage profile}

In this study voltage values were computed for all nodes. However, for the sake of simplicity, mean bus voltage has been reported. Results show that MG also has a positive impact on the voltage profile because the line's current decreases. In turn, this will result in a boost in the voltage at the customer site.

According to Fig. 4, the voltage magnitude increases with MG penetration. Higher load levels lead to higher voltage magnitude increases. This means that the MG benefits have a larger impact on more charged networks. This is particularly important since heavily loaded networks are, naturally, more susceptible to voltage collapse.

In this study, the checkup of voltage bus bars voltage did not detect any violation of limits as a consequence of the growth of MG. On the contrary, increased integration of MG changes some undervoltage states bringing the voltage value into the valid interval, as illustrated by Fig. 4 . As shown, there are some system states for which the voltage is below the minimum for heavy loads and low MG penetration. In such cases, it is clear that increasing the MG penetration level is advantageous.

\subsection{Branches load relief}

The effects of MG on branch load relief are quite dependent of the MG location and magnitude. In some cases, the flow may decrease by $50 \%, 70 \%$ or even more, while in others the line flow might actually increase. This happens when the load is small compared to the MG's generation in that specific network point. This leads to a reversal of the power flow in the branch, which may also increase in magnitude. The former case, when there is a flow decrease, is illustrated in Fig. 5.

Fig. 5 shows the branch flow (as a percentage of its maximum capacity) as a function of MG penetration on two different load instances (C100 and C140). In this example, it was considered that MG units are placed near the loads (FF), which is representative of the large majority of the cases, since MG units are generally placed at the consumer's place of residence (houses, factories, commercial stores). The curves in the graph show that the branch flow decreases when the integration of MG increases, up until very near to $100 \%$.

This behavior, however, does not mean that the MG's impact on the distribution network is negative and MG should be avoided. It should be noted that even for the specific case with $100 \% \mathrm{MG}$ penetration, the branch flow is smaller than for $0 \%$. Furthermore, this occurrence does not represent the most common situation. In 
Flow in Line (12-89) - MG12, FF

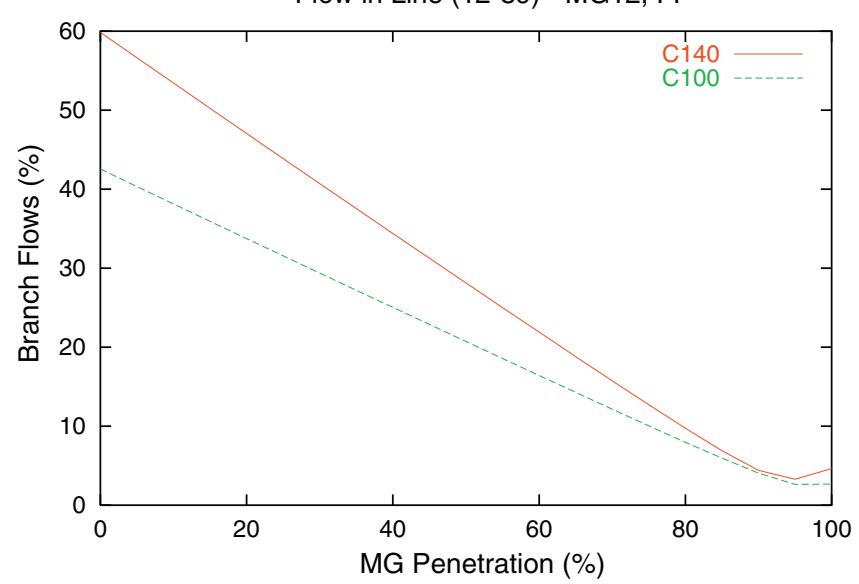

Fig. 5. Effects of the MG penetration level on lines charge.

fact, this situation would be quite rare as it may only happen when the load is quite low and the level of integration of MG is quite high.

Therefore, it can be concluded that in almost all cases MG contributes to a decrease in the power flow in the branches and thus, it typically allows for deferring investment in network capacity.

\subsection{Location effects}

As shown in the previous sections, the location of the MG units does affect the technical impact on the power network, although not inherently. Therefore, in this section its effect is analyzed by comparing its impact on energy losses, voltage profiles and branch loads. Fig. 6 shows the variation of energy loss with MG penetration. The curves are plotted for the six different location scenarios. The graphs show the percentage of energy losses relatively to the case C100 and without MG.

The most unfavorable situation occurs when MG units are near to the feeder, since loss reductions only occur if the levels of penetration of MG are no higher than 30-40\%. For higher penetration levels the losses increase. For the random distribution scenarios (RND1 to RND4) penetration levels of MG up to 50\% are advantageous. Finally, for the scenario where MG is close to the loads (far from the feeder), the loss decreases for the level of penetration of MG up to $60 \%$. These results show that the location of the MG units has an undeniable effect on the power network's technical impact. However, it should be stressed that MG units are most commonly

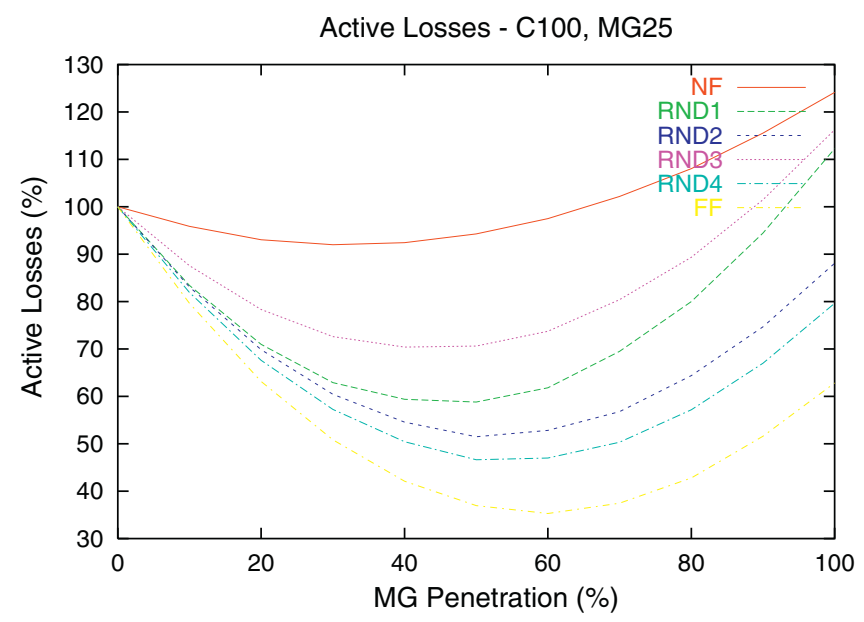

Fig. 6. Effects of the MG location on energy losses.

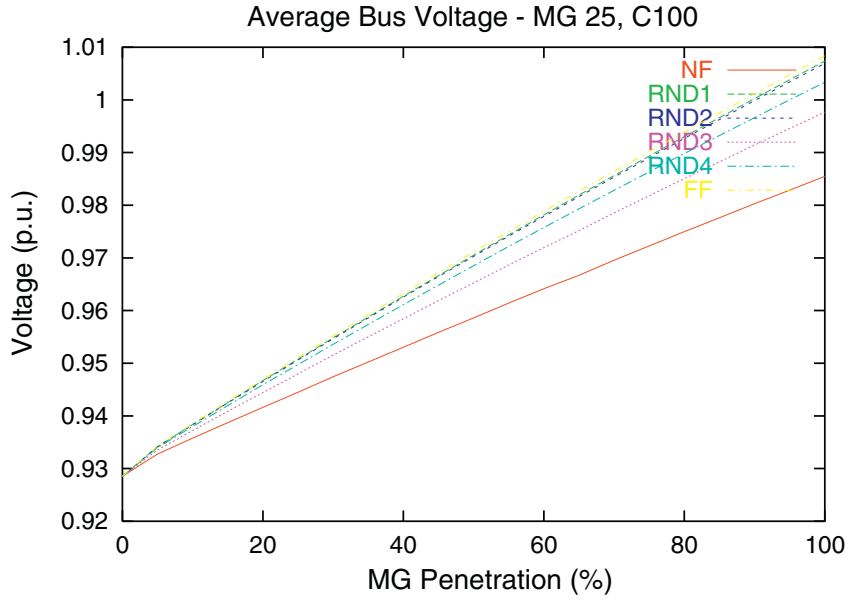

Fig. 7. Effects of the MG location on bus voltage.

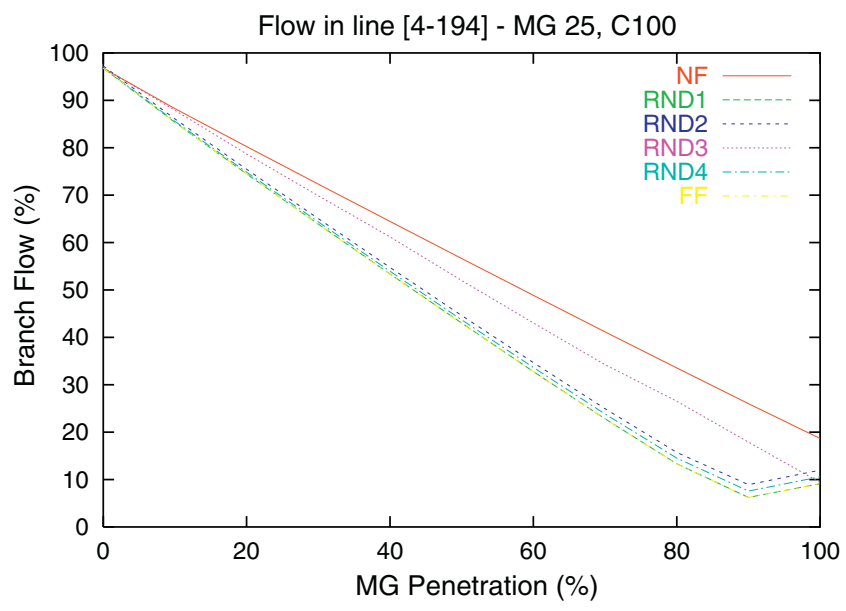

Fig. 8. Effects of the MG location on line charge.

located far from feeders (FF). In fact, the MG units are owned by the consumers and placed near loads (for example at home). Therefore, the general conclusion is that, in the majority of networks, the level of penetration of MG should be allowed to reach up to $50 \%$ or $60 \%$.

Fig. 7 shows the variation of bus voltages as a function of the MG penetration level. As previously, curves are plotted for the six different location scenarios. Once again, it can be seen that the most advantageous location for the MG unit is when it is far away from the feeder (FF), regardless of the level of MG penetration.

The impact of the MG penetration level on line charges is illustrated in Fig. 8. This graph also shows the effect of the six different location scenarios. For the RND1, RND2, and RND4 locations the reduction is the same. This is explained by the fact that, for all cases, no MG unit is connected to the specific branch. In this particular case, FF as well as the RND1, RND2, and RND4 location distributions result in a more intense reduction in the line charge for a penetration level of up to approximately $90 \%$.

\section{Conclusions}

Energy generation from small dispersed MG units can offer a number of interesting advantages for distribution networks. Firstly, systems with MG have several positive technical advantages, such as smaller power losses, better voltage profiles and lower levels of branch flows, which mean investments in line reinforcements can be postponed. Secondly, a considerable part of the total energy is 
produced locally, reducing dependence on fuel imports. Finally, a large majority of the MG units use renewable energy sources and this contributes to creating a cleaner environment by lowering $\mathrm{CO}_{2}$ emissions.

Current legislation should be reviewed and the existing regulatory constraints should be relaxed in order to allow for higher levels of MG penetration. Our study has shown that MG penetration levels of up to at least $50 \%$ have a positive technical impact on the power networks. The current regulatory directive limits the installation of MG to $25 \%$ of the transformer capacity installed in the secondary substation.

In Portugal, the load rarely exceeds $75 \%$ of the capacity of the transformer installed in the secondary substation. Furthermore, the MG's power generation is usually less than its installed capacity - in just $3 \%$ of cases is it above $90 \%$ of its capacity. These factors indicate that the practical regulation limit on the levels of penetration of MG is about $15 \%$.

Moreover, the second legislative constraint on the increase in the penetration of MG, stating that the onsite MG installed capacity should not exceed $50 \%$ of the contracted power for each individual user, further reduces the practical value mentioned above of the penetration of MG.

Although our simulations have shown that some older and longer lines with very high resistance (and also high losses) may experience some voltage problems, from the present study, it is clear that these limitations should be reviewed. Especially, since these types of lines are quite rare in the existing Portuguese distribution system.

\section{References}

[1] T. Beatley, Green Urbanism, Island Press, Washington, DC, 2000.

[2] J. Lopes, Integration of dispersed generation on distribution networks-impact studies, in: Proceedings of Power Engineering Society Winter Meeting, vol. 1, IEEE, 2002, pp. 323-328.

[3] P. Chiradeja, R. Ramakumar, Voltage profile improvement with distributed wind turbine generation-a case study, in: Proceedings of Power Engineering Society General Meeting, vol. 4, IEEE, 2003.

[4] P. Chiradeja, R. Ramakumar, An approach to quantify the technical benefits of distributed generation, IEEE Transactions on Energy Conversion 19 (4) (2004) 764-773.

[5] J. Dolezal, P. Santarius, J. Tlusty, V. Valouch, F. Vybiralik, The effect of dispersed generation on power quality in distribution system, in: Proceedings of Quality and Security of Electric Power Delivery Systems, CIGRE/IEEE PES International Symposium, IEEE, 2003, pp. 204-207.

[6] T. Tran-Quoc, C. Andrieu, N. Hadjsaid, Technical impacts of small dispersed generation units on LV networks, in: Proceedings of Power Engineering Society General Meeting, vol. 4, IEEE, 2003.
[7] Ministério da Economia e Inovação, Publicações e Centros de Documentação, "Política Energética - Vol. II". www.min-economia.pt, 2007 (in Portuguese).

[8] Directive 2006/32 /EC on the energy end use efficiency and energy services and repealing council directive 93/76/EC, Official Journal of the European Union, Strasbourg. www.energv.eu/directives/I11420060427en00640085.pdf, 2006 (online).

[9] R. Burrett, et al. Renewables Global Status Report - 2009 Update, Renewable Energy Policy Network for the 21st Century. http://www.ren21.net/pdf/RE_GSR_2009_Update.pdf, 2009 (online).

[10] John Vidal, http://www.guardian.co.uk/environment/2008/jun/02/ renewableenergy.alternativeenergy (online, cited: 2nd June, 2008)

[11] Micropower Europe, European Policy Update. http:// microgenerationeurope.eu/download/EU_Policy_update_02-2010/pdf, February 2010 (online).

[12] K. Terra, Microgeneration, http://www.kterra.com/kb/Microgeneration22.html (online).

[13] P. Richardson, A. Keane, Impact of high penetrations of micro-generation on low voltage distribution networks, in: 20th International Conference and Exhibition on Electricity Distribution - Part 1, CIRED 2009, 2009, pp. 1-5.

[14] M. Thomson, D.G. Infield, Network powerflow analysis for a high penetration of distributed generation, IEEE Transactions Power Systems 22 (August (3)) (2007) 1157-1162.

[15] A. Beddoes, M. Gosden, I. Povey, The performance of an LV network supplying a cluster of 500 houses each with an installed $1 \mathrm{kWe}$ domestic combined heat and power unit, in: CIRED 19th International Conference on Electricity Distribution, May 2007, 2007 (Paper 0030).

[16] Department of Trade and Industry, UK, PB Power, The Impact of Small Scale Embedded Generation on the Operating Parameters of Distribution Networks. http://www.distributed-generation.gov.uk, 2003.

[17] J.N. Fidalgo, Dalila B.M.M. Fontes, S. Silva, A decision support system to analyze the influence of distributed generation in energy distribution networks, in: J. Kallrath, P.M. Pardalos, S. Rebennack, M. Scheidt (Eds.), Optimization in the Energy Industry, Energy Systems Series, 2009, ISBN: 978-3-540-88964-9, 2008.

[18] P. Moisés Costa, M.A. Matos, J.A. Peças Lopes, Regulation of microgeneration and microgrids, Energy Policy 36 (10) (October 2008) 3893-3904.

[19] N. Silva, G. Strbac, Optimal design policy and strategic investment in distribution networks with distributed generation, in: Seminar SmartGrids for Distribution. IET-CIRED, CIRED, 2008, pp. 1-4.

[20] R.A.C. Van der Veen, L.J. De Vries, The impact of microgeneration upon the Dutch balancing market, Energy Policy 37 (7) (July 2009) 2788-2797.

[21] E. Mashhour, S.M. Moghaddas-Tafreshi, Integration of distributed energy resources into low voltage grid: A market-based multiperiod optimization model, Electric Power Systems Research 80 (April (4)) (2010) 473-480.

[22] A.M. Azmy, I. Erlich, Impact of distributed generation on the stability of electrical power system, in: Power Engineering Society General Meeting, Vol. 2, IEEE, 2005, pp. 1056-1063.

[23] D.G. Infield, J. Short, C. Home, L.L. Freris, Potential for domestic dynamic demand-side management in the UK, in: IEEE Power Engineering Society General Meeting, 2007, 2006, pp. 1-6.

[24] Center for Study of Energy Markets, The Market and Cost of Solar Photovoltaic Electricity Production. http://www.ucei.or, January 2008.

[25] J.D. Harrison, Micro Combined Heat and Power: Potential Impact on the Electricity Supply Industry, http://www.microchap.info, 2001.

[26] Department of Trade and Industry, UK, Mott MacDonald, System Integration of Additional Microgeneration. http://www.distributed-generation.gov.uk, 2004

[27] Sustainable Energy Ireland, PB Power, Cost and benefits of embedded generation in Ireland. http://www.sei.ie, September 2004. 